\title{
Evolving black hole-neutron star binaries in general relativity using pseudospectral and finite difference methods
}

\author{
Matthew D. Duez, ${ }^{1}$ Francois Foucart, ${ }^{1}$ Lawrence E. Kidder, ${ }^{1}$ Harald P. Pfeiffer, ${ }^{2}$ Mark A. Scheel, ${ }^{2}$ and Saul A. Teukolsky ${ }^{1}$ \\ ${ }^{1}$ Center for Radiophysics and Space Research, Cornell University, Ithaca, New York, 14853, USA \\ ${ }^{2}$ Theoretical Astrophysics 130-33, California Institute of Technology, Pasadena, California 91125, USA \\ (Received 29 August 2008; published 13 November 2008)
}

\begin{abstract}
We present a code for solving the coupled Einstein-hydrodynamics equations to evolve relativistic, selfgravitating fluids. The Einstein field equations are solved in generalized harmonic coordinates on one grid using pseudospectral methods, while the fluids are evolved on another grid using shock-capturing finite difference or finite volume techniques. We show that the code accurately evolves equilibrium stars and accretion flows. Then we simulate an equal-mass nonspinning black hole-neutron star binary, evolving through the final four orbits of inspiral, through the merger, to the final stationary black hole. The gravitational waveform can be reliably extracted from the simulation.
\end{abstract}

DOI: 10.1103/PhysRevD.78.104015

PACS numbers: 04.25.dk, 04.30.Db, 04.40.Dg, 47.75.+f

\section{INTRODUCTION}

Compact object binaries containing neutron stars [i.e. neutron star-neutron star (NSNS) and black hole-neutron star (BHNS) binaries] are perhaps as important in modern astrophysics as binary black holes. Both BHNS and NSNS binaries should be excellent sources of gravitational waves for the ground-based interferometric detectors LIGO, GEO, VIRGO, and TAMA. It is possible [1] that the detection rate of these binaries will actually be greater than that of binary black holes. NSNS and BHNS binaries are also interesting because they are leading candidates for explaining the production of short-duration gamma-ray bursts (GRBs) [2], especially given observations that locate some short GRBs in elliptical galaxies [3,4] or rule out an associated supernova [5]. NSNS and BHNS mergers may also be important for understanding the observed abundances of the heavy elements that are formed by rapid neutron capture in the r-process [6].

NSNS and BHNS binary mergers can be accurately modeled only by numerical simulations. In such systems, the spacetime metric and neutron star fluid are both dynamical. They are also strongly coupled, and hence must be evolved simultaneously. In addition, it is clear from the high compactions of the binary objects that only simulations in full general relativity will be adequate. For NSNS binaries, such simulations are particularly needed to study the possible collapse of the post-merger remnant. For BHNS binaries, the crucial questions are (1) whether or not the neutron star is tidally disrupted before it plunges into the black hole, and (2) if the neutron star does disrupt, what fraction of the star's matter is promptly swallowed by the hole, what fraction is ejected, and what fraction forms an accretion disk. To produce a GRB, a substantial accretion disk must remain. To contribute to the abundance of $r-$ process elements, matter must be expelled from the system. Studies strongly suggest that the non-Newtonian form of the gravitational potential [7], gravitational radiation reaction [8], and black hole spin [9] all significantly affect the size of the post-merger accretion disk, underscoring the need for fully relativistic simulations.

Of the two types of systems, NSNS binaries are better studied. Newtonian simulations have been performed using realistic equations of state together with neutrino radiation effects [10-14] and magnetic fields [15]. Simulations using complicated equations of state have also been carried out using the conformally flat approximation to general relativity [16]. General relativistic simulations have been performed using $\Gamma$-law equations of state [17-22] and using more realistic equations of state [23,24]. Recently, general relativistic merger evolutions have been performed that include the neutron star magnetic field $[25,26]$.

Numerical modeling of BHNS binary mergers has been carried out using Newtonian or pseudo-Newtonian gravity (in which the black hole is represented by a point mass) using $\Gamma$-law $[27,28]$ and realistic nuclear $[7,29,30]$ equations of state. BHNS simulations have also been done in conformal gravity [31], although so far only in the extreme mass ratio limit, in which the black hole is much more massive than the neutron star. The first fully relativistic BHNS simulation was of a head-on collision [32]. Most recently, two groups have independently evolved configurations of BHNS binaries in full general relativity from quasicircular inspiral through merger. One group includes Shibata, Taniguchi, Uryū, and Yamamoto [33-35]. The members of the other group are Etienne, Faber, Liu, Shapiro, Taniguchi, and Baumgarte [36]. The evolutions produced by these two groups agree qualitatively, with both now finding very small post-merger disks. However, both groups have so far restricted themselves to $\Gamma=2$ polytropic equations of state and to initially nonspinning black holes. The case of spinning black holes has been studied by Rantsiou, Kobayashi, and Laguna [9] using a Kerr background metric and a Newtonian approximation for the neutron star self-gravity. They find that black hole spin has a strong influence on the size of the post-merger 
accretion disk. For both types of binary, but especially BHNS binaries, the parameter space remains poorly explored. Also, there is an unmet need for results to be checked by multiple independent codes.

In each of the above-mentioned calculations in which the metric variables are truly dynamical fields, these fields were evolved numerically using finite differencing (FD). For a stable evolution, such algorithms should converge to the exact solution as some power of the grid spacing. Also, shock-capturing techniques have been developed which allow FD codes to evolve fluids with discontinuities stably and accurately. FD codes usually require very large grids in order to obtain accurate results, although this problem can be mitigated by using mesh refinement or high-order differencing.

Einstein's equations can also be evolved using spectral methods. In this case, functions are approximated as truncated series expansions in a set of orthogonal basis functions. Derivatives of the approximated functions are then computed exactly. The pseudospectral (PS) method is a type of spectral method that uses the values of functions on a spatial grid of collocation points, rather than directly using the spectral coefficients. This has the advantage that pointwise operations are as straightforward in PS methods as they are in FD methods. In multidomain PS methods, the computational region is divided into domains, each with its own basis functions and corresponding collocation points. For smooth functions, spectral methods (including PS) converge exponentially to the exact solution as the number of basis functions (or, for PS methods, the number of collocation points) is increased. This allows PS methods to get accurate results with much smaller grids than those used by FD codes. A PS code for solving the Einstein equations has been developed by the CornellCaltech relativity group [37-40]. It has been used to simulate the inspiral of binary black holes for many orbits with very high accuracy for a fairly low computational cost [4143].

There is a difficulty, however, in extending PS methods to evolving nonvacuum spacetimes. Because of the presence of stellar surfaces and, in some cases, hydrodynamic shocks, the evolved variables are not smooth in all derivatives. In these cases, spectral representations do not converge exponentially to the exact solution. Rather, they display Gibbs oscillations near the discontinuity that converge away only like some power of the number of collocation points, with the order of convergence given by the order of the discontinuity. The oscillations can be controlled using special forms of filtering or artificial viscosity (e.g. $[44,45]$ ) but exponential convergence is still lost. In some cases, the problem can be avoided by placing domain boundaries at discontinuities, e.g. at the stellar surface. However, this is not practical for complicated shocks or when stars become very deformed (e.g. during tidal disruption).
In this paper, we use the "mixed" approach developed by Dimmelmeier et al. [46] for a conformal gravity code which has been used quite successfully to study supernova core collapse [47-50], and extend it to full general relativity. In this method, the spacetime metric is evolved on one grid using PS methods, the fluid variables are evolved on a second grid using shock-capturing FD or finite volume techniques, and the two grids communicate by interpolation. Below, these two grids are referred to as the "PS grid" and the "fluid grid."

This approach would seem to utilize the strongest features of each method. The spacetime is expected to be smoother than the fluid variables (e.g. at a stellar surface, the discontinuity in metric components appears at a higher derivative than in the density), and so PS techniques should work better on the metric than the fluid. Also, our code uses many spectral domains, and we expect discontinuities to appear in only a few domains. In the domains without discontinuities, the functions can be represented spectrally with the same accuracy as in smooth problems. In the domains with discontinuities, convergence will be limited to a power law. Of course, this error will propagate to the other domains. However, the domain decomposition can often be chosen so that the slower converging domains take up a small fraction of the overall computational region, and their effect on the overall accuracy is correspondingly small. We can also use higher resolution in these domains if the error is still too large. Unlike the strategy of fitting domain boundaries to discontinuities, we do not require the exact locations of discontinuities, but only approximate locations.

Even if the evolution of the fields converges rapidly, spectral accuracy is still lost because of the evolution of the fluids, which will at best converge as a fixed power in the grid spacing on the fluid grid. However, this is not a problem if the resolution on this grid is high. And, in fact, our mixed technique algorithm allows us to achieve high resolution in the hydrodynamic evolution at a surprisingly low computational cost. This is because the fluid grid only needs to cover the region containing the matter. This can provide a huge savings for binary inspiral calculations. For a BHNS inspiral, the fluid grid can be a box centered on the neutron star with outer boundaries slightly outside the star. For a NSNS inspiral, one would need two such boxes. For a BHNS system in which the star is shedding mass onto the black hole, the fluid grid would have to cover a region containing both binary objects, but even then, it would not have to extend all the way out into the wave zone, as it would if the metric were being evolved on the same grid.

In this paper, we describe our code and test its ability to evolve BHNS binaries. In Sec. II, we describe our evolution code. In Sec. III, we present tests of this code. Next, we apply our code to model the inspiral and merger of a BHNS binary. We evolve an equal-mass binary with an 
initially nonspinning black hole and irrotational neutron star. Section IV describes the inspiral calculation, with particular emphasis on the accuracy and rate of convergence of these simulations. Section V describes the merger. Finally, Sec. VI gives our conclusions and future directions for our work.

\section{EVOLUTION CODE}

\section{A. Evolution of the spacetime}

We evolve Einstein's equations using the generalized harmonic formulation [51,52]. We use a first order representation of the system [39], in which the fundamental variables are the spacetime metric $\psi_{a b}$, its spatial first derivatives $\Phi_{i a b}$, and its first derivatives in the direction normal to the slice $\Pi_{a b}$. (Throughout this paper, Latin indices from the first part of the alphabet $a, b, c, \ldots$ run from 0 to 3 , while $i, j, k, \ldots$ run from 1 to 3 .) From these functions, one can easily extract the 3-metric $g_{i j}$, shift $\beta^{i}$, lapse $\alpha$, and extrinsic curvature $K_{i j}$. The evolution of the gauge is determined by the gauge source functions $H_{a}=$ $-\psi^{c d} \Gamma_{a c d}$, which are freely specifiable functions of space and time. The evolution equations for $\psi_{a b}, \Phi_{i a b}$, and $\Pi_{a b}$ are as given in our earlier paper [39], except we add the matter source term

$$
\partial_{t} \Pi_{a b}=\cdots-2 \alpha\left(T_{a b}-\frac{1}{2} \psi_{a b} T^{c d} \psi_{c d}\right) .
$$

We evolve $\psi_{a b}, \Phi_{i a b}$, and $\Pi_{a b}$ using a multidomain PS code described in earlier papers $[37,40,41]$. Each PS domain is either a spherical shell, a cylindrical shell, a cube, a "cubed sphere" (i.e. six cubes distorted so that they fit together to form a sphere [53]), a filled sphere, or a filled cylinder. Spherical harmonics $Y_{l m}$ are used as angular basis functions on spheres. Fourier functions $e^{i m \phi}$ are used for the azimuthal direction on cylinders. Chebyshev polynomials $T_{n}$ are used as basis functions for each direction on cubes and cubed spheres, for the radial direction on spherical shells, and for the radial and $z$-directions on cylindrical shells. The basis functions on filled spheres and cylinders must be chosen specially to have the proper behavior at the origin and axis, respectively. For example, for spheres, we must decompose a function $f$ as $f(r, \theta, \phi)=$ $\sum_{n l m} Q_{n l}(r) Y_{l m}(\theta, \phi)$ (note the coupled indices), where $Q_{n l} \propto r^{l}$ near the origin. We use the functions introduced by Matsushima and Marcus [54] with $\alpha=1, \beta=1$ for filled cylinders and $\alpha=1, \beta=2$ for filled spheres. We use boundary conditions [55] designed to prevent the influx of gravitational radiation and constraint violating metric perturbations. Our domain decomposition is chosen to leave an unfilled (excised) region inside the black hole. No explicit boundary condition need be applied at the boundary of the excised region because all characteristics there flow out of the grid.

The PS grid supplies the fluid grid with the metric fields $\alpha, \beta^{i}, g_{i j}, K_{i j}, \partial_{i} \alpha, \partial_{i} \beta^{j}$, and $\partial_{i} g^{j k}$. These functions must be interpolated from the PS gridpoints to the fluid gridpoints. This could be done directly at each fluid gridpoint by summing the values of all the basis functions at that point with weights given by the known spectral coefficients. This would, however, be prohibitively expensive, as it would involve $\sim N_{\mathrm{FD}} N_{\mathrm{PS}}$ operations, with $N_{\mathrm{FD}}$ the number of destination points (i.e. the number of points on the fluid grid) and $N_{\text {PS }}$ the number of points on a domain of the PS grid. Instead, we used a trick introduced by Boyd [56]. We first interpolate the metric fields onto a finer PS grid. (We usually triple the number of collocation points.) This can be done cheaply by switching to spectral space (often an $N_{\text {PS }} \log N_{\text {PS }}$ operation procedure), adding more basis functions with zero coefficients, and switching back to physical space. Then, one can get an accurate estimate of the field at fluid gridpoints by doing polynomial interpolation from the refined PS grid, which takes only $\sim N_{\mathrm{FD}}$ operations. Using this procedure, the CPU time spent on interpolation, while still significant, is smaller than the time spent on evolving on the PS and fluid grids.

\section{B. Evolution of the hydrodynamic fields}

We model the neutron star matter as a perfect fluid with rest mass density $\rho$, pressure $P$, specific internal energy $\epsilon$, and 4-velocity $u^{a}$, so that the stress tensor is

$$
T_{a b}=\rho h u_{a} u_{b}+P \psi_{a b},
$$

where $h=1+\epsilon+P / \rho$ is the specific enthalpy. The evolution of the fluid is determined by the laws of baryon conservation $\left(\rho u^{a}\right)_{; a}=0$ and energy-momentum conservation $T^{a b}: a=0$. These give five evolution equations for the variables $D=\alpha \sqrt{g} u^{0} \rho, \tau=\alpha^{2} \sqrt{g} T^{00}-D$, and $S_{k}=$ $\alpha \sqrt{g} T^{0}{ }_{k}$. (See Eqs. 2.8-2.10 in [19] for the full evolution equations.) Here $u^{0}$ is given by the normalization condition $\psi_{a b} u^{a} u^{b}=-1$. The pressure is given by the equation of state. Our code is written to evolve general equations of state of the form

$$
\begin{gathered}
\epsilon=\epsilon_{\text {cold }}(\rho)+\epsilon_{\mathrm{th}}, \\
P=P_{\text {cold }}(\rho)+\left(\Gamma_{\mathrm{th}}-1\right) \rho \epsilon_{\mathrm{th}}
\end{gathered}
$$

(cf. [23]). In this paper, we will mainly use the simple $\Gamma$-law equation of state:

$$
\begin{gathered}
P_{\text {cold }}=\kappa \rho^{\Gamma}, \\
\epsilon_{\text {cold }}=P_{\text {cold }} /[\rho(\Gamma-1)], \\
\Gamma_{\text {th }}=\Gamma,
\end{gathered}
$$

which is equivalent to

$$
P=(\Gamma-1) \rho \epsilon .
$$

The hydrodynamic equations have the form

$$
\partial_{t} u+\partial_{i} F^{i}=\mathcal{S}
$$


where $u$ is called a "conservative variable" $\left(D, \tau\right.$, or $S_{k}$, in our case), while $F^{i}$ and $\mathcal{S}$ are the associated flux and source terms, respectively. To solve these equations, one first divides the computational domain into cells, with one cell associated with each gridpoint. Then, from the values of $u$ in the cells, one determines the fluxes $F^{i}$ at the interfaces between cells. From these, one can compute the net flux into each cell, hence obtaining the $\partial_{i} F^{i}$ term in Eq. (9). A specific hydrodynamics evolution algorithm is determined by a set of choices:

(1) Discretization

The values of $u$ at gridpoints can be chosen to be the values of $u$ at the centers of cells (finite difference discretizaton) or to be the average values of $u$ inside the cells (finite volume discretizaton). The distinction only matters for schemes that are higher than second-order accurate. All of our higher order schemes assume finite differencing except for PPM (see below), for which we have coded finite difference and finite volume options. Another discretization issue is the shape of the grid. We always use uniformly spaced Cartesian meshes, i.e., at grid indices $(i, j, k)$ we have gridpoints (cell centers) $\mathbf{x}_{i, j, k}=\left(i \Delta x+x_{0}, j \Delta y+y_{0}, k \Delta z+z_{0}\right)$.

(2) Interpolation variables

To compute the fluxes, five independent variables must be interpolated from cell centers or averages to cell faces. We interpolate $\rho$ to get face densities and $u_{i}$ to get information on face velocities. We have experimented with several choices for the fifth variable, which should carry information about face internal energies. The pressure $P$ is a common choice, and we have found that it works adequately. However, it may not be ideal for evolving lowtemperature stars, the main application of this paper. This is because interpolating $P$ does not separate the zero-temperature component of $P$ from the thermal component. So, even if $P=P_{\text {cold }}(\rho)$ exactly on all grid values, the interpolated $P$ and $\rho$ will not satisfy this relationship because of interpolation error, i.e., there is interpolation "heating." Hence, neutron star simulations carried out using an isentropic equation of state ( $P=\kappa \rho^{\Gamma}$ at all times) can be very accurate for certain problems (e.g. [57,58]). The isentropic treatment completely removes spurious heating, but it is not valid in the presence of shocks.

Another choice would be to evolve $\kappa \equiv P / \rho^{\Gamma}$. For cold polytropes, $\kappa$ is a constant and can be interpolated exactly. The pressure at faces is then constructed from the interpolated $\kappa$ and interpolated $\rho$. We find that interpolating $\kappa$ works well for cold stars, but behaves badly at shocks. This can be fixed by switching the scheme at discontinuities back to interpolating $P$. Even so, its usefulness is limited to polytropes. An even better choice is to interpolate
$P_{\text {th }} \equiv P-P_{\text {cold }}$, the thermal part of the pressure. Then the pressure on the face is the sum of the interpolated $P_{\text {th }}$ and the $P_{\text {cold }}$ computed from the interpolated $\rho$. With this choice, interpolation error introduces no heat on the faces for a zerotemperature star. In addition, it seems to have no problems evolving shocks, and it can be used with any equation of state. By interpolating either $\kappa$ or $P_{\text {th }}$, we find that spurious heating in our stars is often significantly reduced.

(3) Interpolation method

Interpolation must be done in a special way to accommodate the possibility of shocks. (The process is usually called "reconstruction" in the literature.) Consider a one-dimensional problem, in which we know grid values $p_{i}$ of the function $p(x)$. The reconstruction step involves computing $p_{L}=p_{i-1 / 2-\epsilon}$ and $p_{R}=p_{i-1 / 2+\epsilon}$, i.e. the values of $p$ to the left and right of the grid cell interface. (In three dimensions, one must reconstruct at faces in each direction.) Many techniques have been developed for interpolating from cell centers or averages to faces. We have implemented second-order monotonized central (MC) reconstruction, third-order piecewise parabolic (PPM) [59] reconstruction, third-order convex essentially nonoscillatory (CENO) [60] reconstruction, and third-order weighted essentially nonoscillatory (WENO) [61] reconstruction. We have found that finite volume PPM usually gives the best results when evolving equilibrium stars. We have checked that this scheme achieves third-order convergence for onedimensional smooth flows. For more general problems, we usually find second-order convergence. (See tests below.)

(4) Flux computation

From $p_{R}$ and $p_{L}$, one can compute $u_{R}$ and $u_{L}$, the face values of the conservative variables, and $F_{R}$ and $F_{L}$, the face values of the fluxes. The "true" flux at the interface must be constructed by some sort of combination of the values on the two sides. We use the scheme of Harten, Lax, and van Leer (HLL) [62], in which the flux is

$$
F_{i-1 / 2}=\frac{c_{\min } F_{R}+c_{\max } F_{L}-c_{\min } c_{\max }\left(u_{R}-u_{L}\right)}{c_{\min }+c_{\max }},
$$

where $c_{\min }$ and $c_{\max }$ are the left-going and rightgoing sound speeds. The first two terms in Eq. (10) provide an average value of the flux, while the last two contribute to a stabilizing diffusive term.

The time derivative of the variable $u$ is equal to $\mathcal{S}-$ $\operatorname{div} F$ [cf. Eq. (9)]. For a finite volume method, the flux divergence is just 


$$
\operatorname{div} F_{i}=\frac{F_{i+1 / 2}-F_{i-1 / 2}}{\Delta x}
$$

plus the equivalent terms for the other two dimensions. For a finite difference method, other terms must be added to achieve third-order accuracy (see e.g. [63] for details).

The vacuum region outside the star or stars requires special treatment. We fill the vacuum region on the fluid grid with a very low density $\left(10^{-7}\right.$ of the maximum value of $\rho$ ) atmosphere, as is usually done for these types of problems. At each step, we apply a floor on $\rho$. We also apply both a floor and a ceiling on the temperature in the atmosphere, requiring $0<T<8\left(P_{\text {cold }} / \rho\right)$. Finally, we limit the velocity in the atmosphere to some fraction (e.g. 0.8 ) of the speed of light. The pressure and velocity cutoffs are only applied in regions where the density is below a chosen threshold (usually $\sim 10^{-3}$ of the maximum value of $\rho$ ). Therefore, they should have little effect on evolutions.

The PS grid requires $T_{a b}$ at each collocation point, so we must interpolate from the fluid grid to the PS grid. We have written an arbitrary-order polynomial interpolator and a third-order WENO interpolator. One would expect the latter to give more sensible results near strong shocks, but for the applications reported here, both work sufficiently well.

When evolving black hole systems, we excise the gridpoints in a neighborhood of the singularity and evolve the region near the excision zone using one-sided differencing as described in [64]. Of course, the excised region of the fluid grid must match that of the PS grid. Also, when interpolating from the fluid to the PS grid, we choose interpolation stencils which avoid the excised region.

When symmetries are present in a problem, they can sometimes be used to reduce the size of the fluid grid. We have coded options to impose reflection symmetries on the $x=0, y=0$, or $z=0$ planes, or any combination of them. In these cases, the fluid grid covers only part of the system, with fluid quantities in other regions being filled for the PS grid using the appropriate symmetries. We have also coded a version of the hydrodynamics code that assumes axisymmetry and uses a two-dimensional fluid grid.

Both the PS and the hydrodynamics modules of our code use multiple domains. This allows the code to be run in parallel by assigning different domains to different processors. We place ghost zones at processor interfaces in the fluid grid, so that numerical derivatives can be correctly taken at all true gridpoints.

\section{Evolution of the coordinates}

Accuracy in a multidomain PS code strongly depends on having a domain decomposition which matches the "shape" of the fields being evolved. Also, the excised region of a black hole must remain inside the horizon. Thus, it is desirable for the grid to move with the black holes and neutron stars. We do this using the "dual coordinate frames" system developed for binary black hole evolutions [41]. The coordinate frame $x^{\bar{l}}$ is set to be an asymptotically flat, inertial frame. All tensor components are evaluated with respect to this frame. The PS and fluid gridpoints are fixed in the computational frame $x^{i}$. By means of a mapping between the frames, the computational coordinates approximately comove with the system. For example, one can track a binary using a simple combination of rotation and radial scaling:

$$
\begin{aligned}
\bar{t} & =\bar{t}, \\
\bar{x} & =x_{c}+a\left[\left(x-x_{c}\right) \cos (\Phi)-\left(y-y_{c}\right) \sin (\Phi)\right], \\
\bar{y} & =y_{c}+a\left[\left(x-x_{c}\right) \sin (\Phi)+\left(y-y_{c}\right) \cos (\Phi)\right], \\
\bar{z} & =a z,
\end{aligned}
$$

where $\left(x_{c}, y_{c}, 0\right)$ is the point at which $x^{i}=x^{\bar{l}}$, which we choose to be fixed for all time, while $\Phi$ and $a$ are functions of time. One evolves $\Phi$ to correct for the binary's orbital rotation, and $a$ to correct for any change in the orbital separation (i.e. inspiral or eccentricity). This is done by introducing a feedback mechanism into the evolution of $\Phi$ and $a$ (see [41] for details).

The dual frame mappings actually used in our simulations are very similar to Eq. (12) with one notable difference. For large outer radius $R_{0}$, small changes in $a$ lead to large absolute changes in $a R_{0}$, causing an instability. Therefore, we replace the linear scaling $\bar{r}=$ ar used in Eq. (12) with $\bar{r}=a r+(1-a) r^{3} / R_{0}^{2}$, so that we recover the linear scaling when $r \ll R_{0}$, but the outer boundary is fixed even when $a$ changes. Such a mapping has been successfully used for long-inspiral binary black hole simulations [43]. Hereafter, we will refer to this coordinate map, a rotation combined with a nonlinear scaling, as $\mathcal{R} \mathcal{S}$.

Since the equations we integrate are written in the inertial frame, they involve derivatives with respect to $x^{\bar{l}}$. These derivatives are obtained by first taking derivatives with respect to grid coordinates $x^{i}$ and then transforming them using a Jacobian matrix. So, for example, the equation

$$
\partial_{\bar{t}} u^{\bar{\alpha}}+A^{\overline{k \alpha}}{ }_{\bar{\beta}} \partial_{\bar{k}} u^{\bar{\beta}}=0
$$

becomes

$$
\partial_{t} u^{\bar{\alpha}}+\left[\frac{\partial x^{i}}{\partial \bar{t}} \delta_{\bar{\beta}}^{\bar{\alpha}}+\frac{\partial x^{i}}{\partial x^{\bar{k}}} A_{\bar{\beta}}^{\overline{k \alpha}}\right] \partial_{i} u^{\bar{\beta}}=0 .
$$

Both the metric and the fluid can be evolved in this way. Thus Eq. (9) becomes

$$
\partial_{t} u+\frac{\partial x^{i}}{\partial x^{\bar{k}}}\left[\partial_{i}\left(\frac{\partial x^{i}}{\partial \bar{t}} \frac{\partial x^{\bar{k}}}{\partial x^{i}} u+F^{\bar{k}}\right)-u \partial_{i}\left(\frac{\partial x^{i}}{\partial \bar{t}} \frac{\partial x^{\bar{k}}}{\partial x^{i}}\right)\right]=\mathcal{S} .
$$

Note that we have moved the coordinate advection term $\frac{\partial x^{\bar{k}}}{\partial x^{i}} \frac{\partial x^{i}}{\partial \bar{t}} u$ into the derivative containing the flux so it can mostly cancel the velocity advection term in $F^{\bar{k}}$. 
There are some subtleties involved in applying dual frames to our shock-capturing techniques; a straightforward evolution of Eq. (15) will often be unstable. However, we have implemented two techniques for evolving fluids in moving coordinates that have proven to be robustly stable. We believe that the advantages of moving coordinates are so great that it is useful to report both methods.

The conceptually simpler technique is to transform the metric and the field variables into moving coordinates, and then evolve as usual in this coordinate system. One must remember that $D, \tau$, and $S_{k}$ are densities, so transforming them to the moving frame involves multiplying by $\operatorname{det}\left(\partial x^{i} / \partial x^{\bar{k}}\right)$. Since the spectral code evolves tensors using inertial frame components, coordinate transformations must be performed when the two grids communicate.

It is also possible to evolve the hydrodynamic equations with inertial frame components using Eq. (15). However, one must make a few adjustments to the shock-capturing code. (1) The $c_{\min }$ and $c_{\max }$ in Eq. (10) should be computed in the moving frame. (2) The fluxes in Eq. (10) must be broken up into two pieces. For example, for the $x$-interface fluxes

$$
\begin{aligned}
& F_{i+1 / 2}^{\bar{k}(1)}=\left(c_{\min }+c_{\max }\right)^{-1}\left(c_{\min } F_{R}^{\bar{k}}+c_{\max } F_{L}^{\bar{k}}\right), \\
& F_{i+1 / 2}^{x(2)}=\left(c_{\min }+c_{\max }\right)^{-1} c_{\min } c_{\max }\left(u_{R}-u_{L}\right) .
\end{aligned}
$$

The $\bar{k}$ index may seem unfamiliar; it is not usually needed because in evolutions without dual frames, one only needs the $x$-derivative of $F^{x}$, the $y$-derivative of $F^{y}$, and the $z$-derivative of $F^{z}$. However, the Jacobian in Eq. (15) mixes derivatives, so the other derivatives are now needed. To the two flux pieces correspond two pieces of the flux derivatives

$$
\begin{aligned}
& \left(\partial_{x} F^{\bar{k}}\right)_{i}^{(1)}=\Delta x^{-1}\left(F_{i+1 / 2}^{\bar{k}(1)}-F_{i-1 / 2}^{\bar{k}(1)}\right), \\
& \left(\partial_{x} F^{x}\right)_{i}^{(2)}=\Delta x^{-1}\left(F_{i+1 / 2}^{x(2)}-F_{i-1 / 2}^{x(2)}\right),
\end{aligned}
$$

and similarly for $y$ and $z$. Next, the Jacobian in Eq. (15) is applied to $\left(\partial_{i} F^{\bar{k}}\right)^{(1)}$ to give $\left(\partial_{\bar{i}} F^{\bar{k}}\right)^{(1)}$. The Jacobian is not applied to $\left(\partial_{i} F\right)^{i(2)}$; this term is added directly to the time derivative of $u$. If the Jacobian is applied to $\left(\partial_{i} F\right)^{i(2)}$, it will not behave correctly as a diffusion term, and the code will be unstable.

\section{CODE TESTS}

\section{A. Shocks}

As a first test of the hydrodynamics code, we perform a standard Riemann shock tube problem. We evolve a $\Gamma=$ $5 / 3$ fluid with an interface separating regions of different $\rho$ and $P$. The initial state is $(\rho, P, v)=(10,13.3,0)$ on one side of the interface and $(\rho, P, v)=(1,0,0)$ on the other. The interface is chosen to lie along the $x y$ diagonal, so the problem is numerically two dimensional. We evolve using the PPM reconstructor on a grid of $150^{2}$ points and grid spacing $\Delta x=0.013$. The results at $t=0.35$ are shown in Fig. 1. We see that our code captures all of the features of the exact solution.

\section{B. Spherical accretion}

Next, we check that our code can accurately model accretion onto a nonrotating black hole. Note that accretion test problems provide very sensitive checks of a code's treatment of the effects of curved spacetime on the fluid flow (the non-Minkowski pieces of the fluid equations), and also checks the code's ability to advect matter through an excision boundary without losing stability or accuracy.

We perform three types of accretion tests. For the first test, we check that the code can maintain an equilibrium radial accretion flow. As an initial state, we use the wellknown exact solution of Michel [65]. We use Kerr-Schild coordinates and excise all gridpoints inside a radius of $r=$ $1.6 M$, where $M$ is the mass of the black hole, so the event horizon is at $r=2 M$. We choose a $\Gamma=5 / 3$ fluid and a flow with the sonic point at $r_{s}=8 M$. As an outer boundary condition, we hold the fluid variables at $r>12 M$ to the exact values. The metric fields are held fixed-otherwise, the black hole would grow, and the problem would not be stationary. Derivatives of the metric are computed analytically. The fluid is evolved to $t=100 M$ on three grids, with grid spacings of $\Delta x=0.3 M, \Delta x=0.2 M$, and $\Delta x=$ $0.15 M$. We assume that octant symmetry is maintained, and so evolve only one octant. The results are shown in the top panel of Fig. 2. The solution remains accurate everywhere, including near the sonic point. We find slightly worse than second-order convergence. This is because of the nonsmooth flow at the sonic point. We find that in most of the smooth regions, the flow converges to the exact solution at second order.

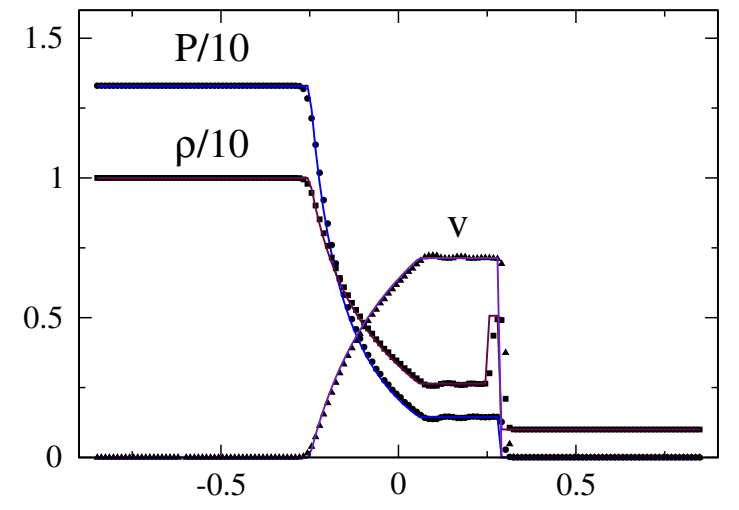

FIG. 1 (color online). A snapshot at $t=0.35$ of the density, pressure, and 3-velocity at each gridpoint on a line perpendicular to the shock interface. The lines show the exact solution. Circles, squares, and triangles show the numerical pressure, density, and velocity, respectively. 


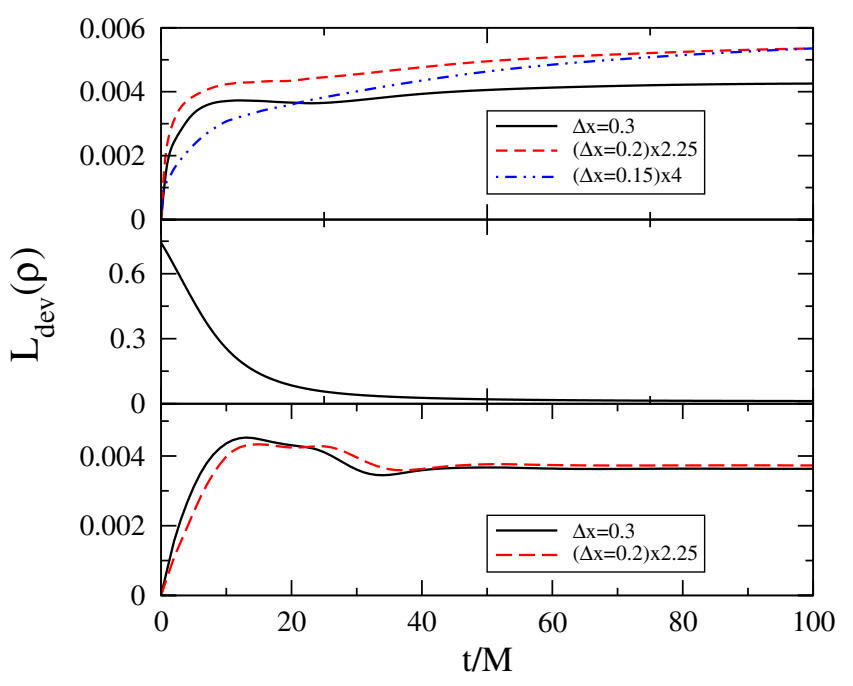

FIG. 2 (color online). The deviation of the density $\rho$ from its exact equilibrium $\rho_{\mathrm{ex}} \cdot L_{\mathrm{dev}}(f)$ is a relative L2 norm: $L_{\mathrm{dev}}(f)=$ $L 2\left(f-f_{\text {ex }}\right) / L 2\left(f_{\text {ex }}\right)$, where the norms sum only over points outside the horizon. The top panel is a relativistic Bondi flow, the middle panel is a perturbed Bondi flow, and the bottom panel is a moving black hole flow. The deviations in the top and bottom panels are scaled for second-order convergence.

For the second test problem, we perturb the initial state by multiplying the density by $1-e^{-[r /(6 M)]^{3}}$. We then evolve with the same outer boundary condition as before. The flow should relax to the known equilibrium solution, and one can see from the middle panel of Fig. 2 that it does.

Finally, we simulate a case in which the black hole is moving relative to the fluid at large distance. We choose the fluid to have the equation of state $P=\rho$. For this equation of state, the equilibrium has been computed analytically by Petrich, Shapiro, and Teukolsky [66]. We take the relative velocity between the hole and the distant fluid to be $0.6 c$ in the $z$-direction, and we evolve in the black hole's frame. The fluid is evolved at two resolutions, corresponding to $\Delta x=0.3 M$ and $\Delta x=0.2 M$. Because of the front-back asymmetry, we must evolve two octants. The result is shown in the bottom panel of Fig. 2. The convergence is second order. We note that for this equation of state, the speed of sound is equal to the speed of light, so there is no sonic point, and the solution is smooth everywhere.

\section{Equilibrium relativistic stars}

In the previous tests, the matter flow did not affect the spacetime metric. For our next test, we evolve equilibrium relativistic stars. Self-gravity is obviously of fundamental importance for such objects, so the fluid and spacetime should now be evolved together, and we expect significant feedback in both directions. For this test, we evolve isolated stars with polytropic equation of state $P=\kappa_{0} \rho^{1+1 / n}$, where we choose $n=1$. We choose units such that $G=$ $c=\kappa_{0}=1$. In these units, the maximum mass for a non- rotating $n=1$ polytrope is $M_{\operatorname{maxTOV}}=0.164$. This is the mass of a polytrope that has the critical central density $\rho_{\text {crit }}=0.32$. Equilibrium configurations for rotating stars were computed using the code of Cook, Shapiro, and Teukolsky [67].

We study four stars, whose properties are summarized in Table I. Stars A and B are nonrotating. Star A has central density $(2 / 3) \rho_{\text {crit }}$ and star B has central density $(4 / 3) \rho_{\text {crit }}$. From the turning point theorem [68], we infer that star $\mathrm{A}$ is stable and star B is unstable. These stars have octant symmetry and are evolved using fluid grids that cover one octant. Stars C and D are rotating, and we evolve them using fluid grids that cover the upper half-plane. Star C has the same rest mass as star A, but it rotates rigidly at an angular speed of $70 \%$ its mass shedding limit. From the turning point theorem, we know this star is secularly stable, and we expect it to be dynamically stable as well. Star D is a differentially rotating, hypermassive star similar to one studied by other groups $[69,70]$. It is known from the simulations of these groups to be dynamically stable.

First, we investigate the convergence of the fluid and PS codes, and, in particular, the effect of the discontinuity in the gradient of $\rho$ on the convergence of the PS code. For this, it is sufficient to look at the spherically symmetric star A configuration. This star has a nonzero density at any point whose coordinate distance $r$ from the star's center is less than the stellar radius $R$ in these coordinates.

We carry out three sets of convergence tests, with results shown in Fig. 3. First, we hold the metric fixed and evolve the fluid using three resolutions of the fluid grid. In these tests, the fluid grid is a cube covering one octant which extends from the origin to $1.3 R$ along each axis. As expected, we see second-order convergence. Because we plot the L2 norm of the deviation of $\rho$ from its initial value, the frequency of oscillations seen in the plot is twice the fundamental radial mode frequency. Not surprisingly, we find similar convergence to the exact stationary solution using the axisymmetric fluid code.

Next, we hold the hydrodynamic variables fixed and evolve the metric using the PS code. We fix the generalized

TABLE I. For each of the equilibrium polytropic stars used to test the code, the ratio of the ADM mass $M$ to the TOV maximum mass $M_{\text {maxTov }}$, the ratio of the polar $\left(R_{\text {polar }}\right)$ to the equatorial $\left(R_{\text {equat }}\right)$ coordinate radius, the ratio of the rotational kinetic energy $T$ to the gravitational potential energy $|W|$, and the ratio of the angular velocity on the equator $\Omega_{\text {equat }}$ to the angular velocity on the rotation axis $\Omega_{\text {axis }}$.

\begin{tabular}{lcccc}
\hline \hline Star & $M / M_{\text {maxTOV }}$ & $R_{\text {polar }} / R_{\text {equat }}$ & $T /|W|$ & $\Omega_{\text {equat }} / \Omega_{\text {axis }}$ \\
\hline A & 0.96 & 1.00 & 0.00 & $\mathrm{NA}$ \\
B & 0.99 & 1.00 & 0.00 & NA \\
C & 0.96 & 0.75 & 0.06 & 1.0 \\
D & 1.70 & 0.30 & 0.25 & 0.3 \\
\hline \hline
\end{tabular}




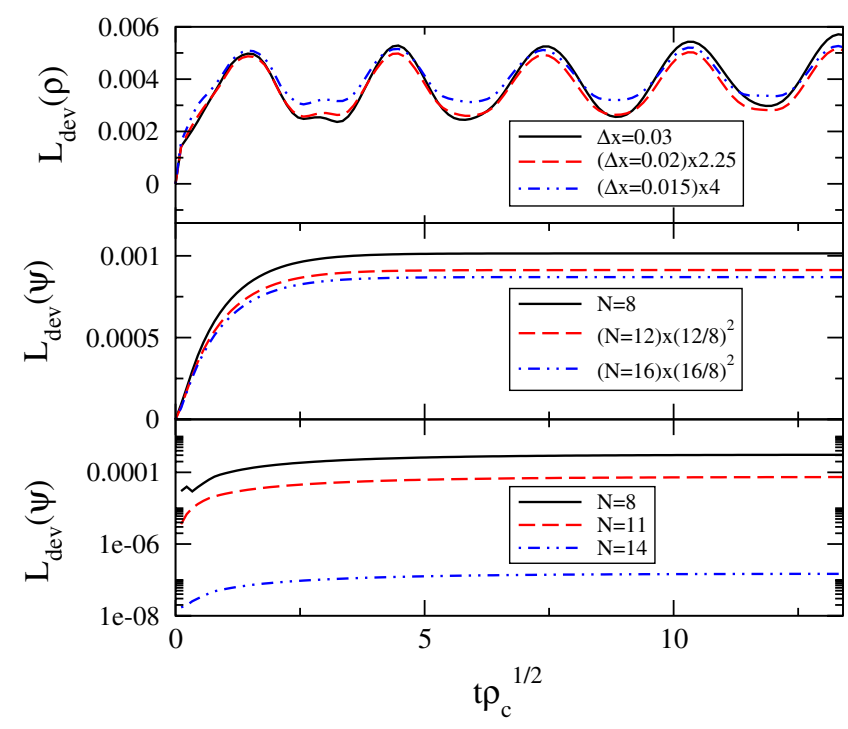

FIG. 3 (color online). The deviations from known equilibrium values of the density $\rho$ and 4-metric $\psi_{\alpha \beta}$ for various grids. As in Fig. $2, L_{\mathrm{dev}}(f)=L 2\left(f-f_{\mathrm{ex}}\right) / L 2\left(f_{\mathrm{ex}}\right)$. In the top panel, the metric is fixed and the fluid is evolved. In the other panels, the fluid is fixed and the metric is evolved. In the middle panel, the computational domain contains the stellar surface. In the bottom panel, the domain is entirely inside the star.

harmonic gauge source functions $H_{a}$ at their initial values, which are chosen to make the spacetime stationary in our coordinates. To study the effects of the surface, we do two sets of runs. First, we evolve the metric on a domain that contains the stellar surface in the domain interior. The domain chosen is a spherical shell with inner radius at $0.7 R$ and outer radius at $1.3 R$. The middle panel of Fig. 3 shows the results for PS grids with 8,12 , and 16 radial collocation points. The nonsmoothness of the metric at the surface reduces the convergence to second order.

Finally, we evolve the metric in a PS domain in the interior of the star. The domain chosen is a cube at the center of the star with sides of length $0.86 R$. We evolve with 8,11 , and 14 collocation points in each direction. The resulting error is plotted in the bottom panel of Fig. 3. The error should decrease exponentially as the number of points is increased, and we do see extremely rapid convergence.

To test the full code, we next evolve a star in time, allowing both the hydrodynamic fields and the metric to change and influence each other. First, we check that the code still converges. To demonstrate the generality of the hydrodynamics code, we pick a more complicated equation of state for the star used in this test. (Of course, we have also checked that this test works with the polytrope star A.) We choose the SLy neutron star equation of state [71] to describe $P_{\text {cold }}$, using the fitting function approximation presented in [23], and we choose $\Gamma_{\mathrm{th}}=2$. The star has a central density of $10^{15} \mathrm{~g} \mathrm{~cm}^{-3}$ and a mass of $1.4 M_{\odot}$. In Fig. 4, we show results from evolving this star at two

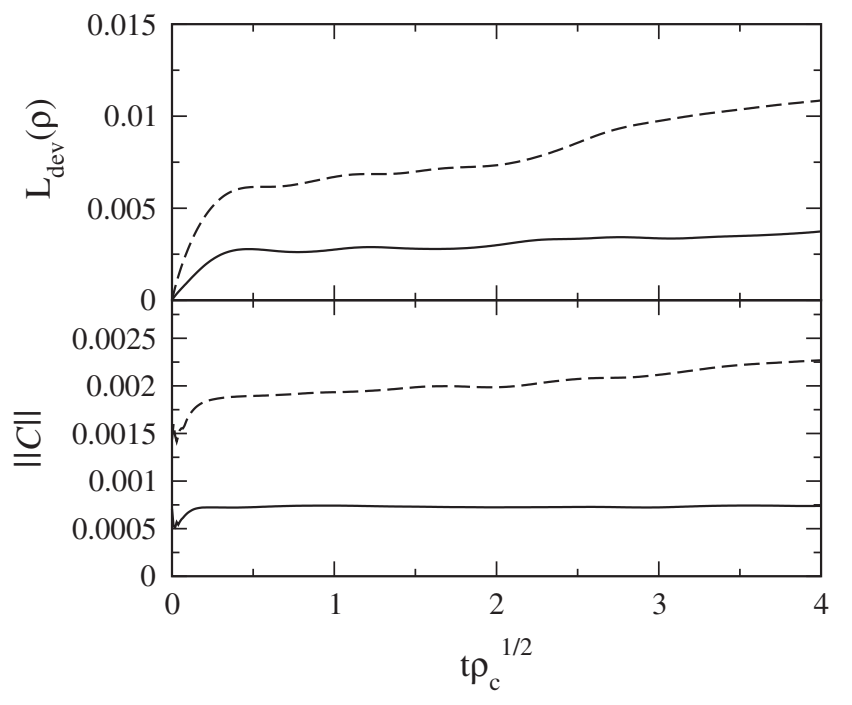

FIG. 4. A neutron star with a realistic equation of state with both matter and metric evolved. Two runs are shown, corresponding to lower resolution PS and fluid grids and higher resolution grids. We plot the normalized L2 norm of the change in density and the normalized L2 norm of the violation in the generalized harmonic constraints.

resolutions. In the low resolution run, the fluid grid has $16^{3}$ gridpoints covering one octant, while the PS grid is an $11^{3}$ cube surrounded by 7 spherical shells, each with 6 radial collocation points and a maximum angular $l$ of 8 . In the high resolution run, the fluid grid has $24^{3}$ points, while for the PS grid we add one basis function in each direction in each domain. Figure 4 shows the deviation of the density from its initial profile and the violation in the generalized harmonic constraint equations, the latter measured by the normalized constraint energy $\|\mathcal{C}\|$ defined by Eq. (71) in [39]. We see that both measures of error decrease significantly as resolution is increased. (In this case, we have no expected convergence rate for comparison. However, we note that in Fig. $4, L_{\mathrm{dev}}(\rho)$ decreases as if it were converging to second order in fluid grid spacing.)

As a final test, we evolve all of the polytropic stars (A, B, $\mathrm{C}$, and D) for many dynamical times to check that our code can distinguish stable from unstable stars and can evolve stable stars accurately for long times. We evolve stars A and $\mathrm{B}$ using octant symmetry to restrict the fluid evolution to a single octant. For these stars, we divide the computational domain into 8 fluid domains with $18^{3}$ gridpoints each and 8 PS domains with roughly $10^{3}$ collocation points on an average domain. For stars $\mathrm{C}$ and $\mathrm{D}$, we utilize equatorial symmetry to restrict the fluid evolution to the upper halfplane. We use 32 fluid domains of $19^{3}$ gridpoints and 32 PS domains of roughly $10^{3}$ points each. For all four stars, the PS grid consists of a cube at the star's center, a spherical shell well outside the star at the outer boundary, and the region in between covered by several layers of cubed spheres, one of which contains the stellar surface. The 


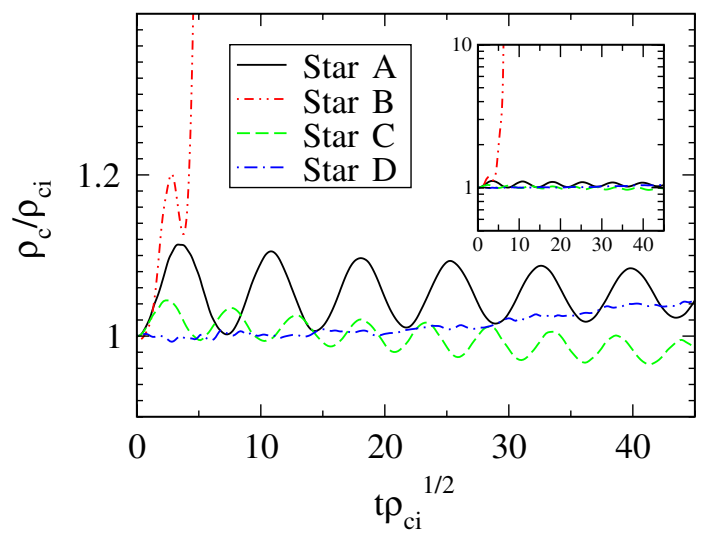

FIG. 5 (color online). The central density (relative to its initial value) for simulations of the four isolated stars. At the initial time, each star is subjected to a perturbation in the form of a $1 \%$ pressure depletion throughout the star. The inset shows the same thing but with a larger density range, so that later stages of the collapse of star B can be seen.

generalized harmonic gauge source functions $H_{a}$ are again chosen to be fixed in time at their initial values. In order to test the stability of each star, we perturb the initial data by reducing the pressure everywhere by $1 \%$. The induced violation of the constraint equations is small, so we do not adjust the metric to re-solve the constraints.

In Fig. 5, we plot the central density as a function of time for each star. As anticipated, stars A, C, and D are stable, while star B collapses. (We do not attempt to follow the collapse of star B to late times because we expect our choice of $H_{a}$ to be poor once the density profile changes drastically.) We see that the stable stars remain close to their initial states for many dynamical times. (For example, we have evolved the hypermassive star D for 20 central rotation periods.) Of the stable stars, star A has density oscillations with the highest amplitude, probably because its central density is close to $\rho_{\text {crit }}$. The oscillations have a period of 16.7, which is close to the expected period for linear radial oscillations of $\sim 7 \rho_{c}^{-1 / 2}=15.7$.

\section{BLACK HOLE-NEUTRON STAR BINARY INSPIRAL}

We now apply our code to model a BHNS binary, starting from the late stage of its inspiral. To date, there have been only a few successful simulations of these important systems in full general relativity [33-36]. For this paper, we have chosen to evolve an equal-mass system. Of course, we do not expect such a system to occur frequently in nature, but we found this choice useful for two reasons. First, it allows us to make maximum use of our experience with binary black holes, which has largely focused on the equal-mass case. Second, since some of the most important work on BHNS binaries with relativistic gravity (e.g. [31]) has been done in the limit of extreme mass ratios, an equal-mass system allows us to emphasize that we do not have this restriction.

The code used to construct our initial data is described in [72]. For simplicity, and to facilitate comparisons with the results of other codes, we have modeled the neutron star as a $\Gamma=2$ polytrope. At the start of the evolution, the neutron star is chosen to be irrotational, and the black hole is chosen to have a zero quasilocal spin. (See [72,73] for details.) We use units such that the mass of the black hole and the neutron star are both one. (We define the mass of the star to be the Arnowitt-Deser-Misner (ADM) mass of an isolated star with the same baryonic mass as the one in the binary. Because the black hole has vanishing spin, we simply use the irreducible mass of the apparent horizon as the black hole mass.) At the initial time, we place the black hole and neutron star in nearly circular orbit with a binary separation of 24 in our coordinates and units. In particular, we place the center of rotation at the point $(0,0,0)$, the maximum density inside the star at $(-12,0,0)$, and the center of the horizon at $(12.011,0.097,0)$. The rotation axis is the $z$-axis, and the initial period is $P=580$. At this separation, the binary is expected to evolve a few orbits before the neutron star is disrupted. As we evolve, we use our dual frame system to hold the center of mass of the star (which nearly coincides with its maximum density point) at $(-12,0,0)$ in moving coordinates, and we drive the horizon center to $(12.011,0,0)$. After a quick adjustment, in which the black hole center moves onto $y=0$, we find that the centers remain locked throughout the evolution. The coordinate mapping used to track the binary objects is a combination of two maps of type $\mathcal{R S}$ described in Sec. II C. One $\mathcal{R} \mathcal{S}$, labeled $\mathcal{R} \mathcal{S}^{1}$, is chosen to have a rotation axis that runs through the origin, and its $a$ and $\Phi$ parameters are used to fix the $x$ and $y$ moving coordinates of the center of the apparent horizon. If the two binary objects were identical (as in the equal-mass, nonspinning binary black hole problem), this one mapping would fix both objects by symmetry. In our case, however, the black hole and neutron star are not identical and asymmetries may develop. So we introduce a second mapping. The second $\mathcal{R S}$, labeled $\mathcal{R} \mathcal{S}^{2}$ is chosen to have a rotation axis at $x=12.011, y=0$, the location of the black hole. This map does not affect the location of the black hole, but its $a$ and $\Phi$ are adjusted to fix the neutron star center of mass, defined as $\int x^{i} D d^{3} x / \int D d^{3} x$. In the case reported here, the second map is nearly an identity and so is not really needed.

The fluid grid is chosen to be a cube of side length 16 . (The diameter of the star is 11.5.) The pseudospectral grid consists of 7 cubes, 22 shells, 28 cylinders, and one cubed sphere. It extends to an outer radius of 326. In order to test the accuracy and convergence of the inspiral simulation, we vary the resolutions of both grids as shown in Fig. 6. We evolve with three fluid grids, having $48^{3} \times \frac{1}{2}, 72^{3} \times \frac{1}{2}$, $96^{3} \times \frac{1}{2}$ total gridpoints. (The $\frac{1}{2}$ factors come from our 


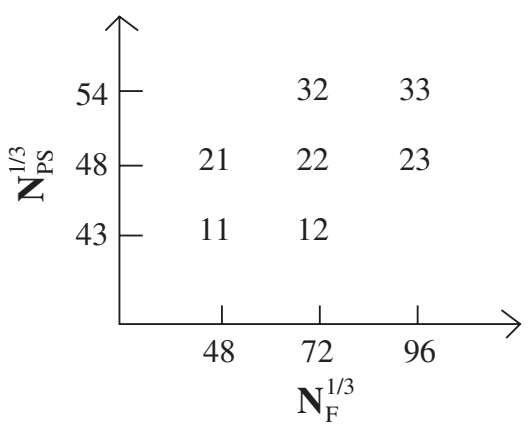

FIG. 6. A representation of the choices of grid resolutions used in our reported runs. $N_{F}$ is the effective total number of grid points on the fluid finite difference grid, which includes "mirror" points given by the reflection symmetry about the equatorial plane; $N_{\mathrm{PS}}$ is the total number of collocation points on the spectral grid.

use of equatorial symmetry to evolve only above the equator.) We also use three PS grids, with $43^{3}, 48^{3}$, and $54^{3}$ collocation points. The $43^{3}$ and the $48^{3}$ grids (and also the $48^{3}$ and $54^{3}$ grids) differ by the addition of one collocation point (i.e. one new basis function) in each direction on each domain, with the exception that the azimuthal direction on cylinders is incremented by two points (which corresponds to adding a new sine and cosine basis function), as is needed to see convergence. To test boundary effects, the middle resolution (22 in Fig. 6) is also run on a pseudospectral grid with outer radius of 400 .

Each resolution is run on 32 processors on the Caltech SHC cluster. For resolution 22, it takes 37 hours (1184 CPU hours) to evolve one orbit at the initial separation. At resolution 33, it takes 100 hours (3200 CPU hours) to go one orbit.

We evolve to $t=1000$ (a little under two periods) at each resolution. The results are shown in Figs. 7-10. In Fig. 7, we plot the normalized constraint energy $\|\mathcal{C}\|$ as a

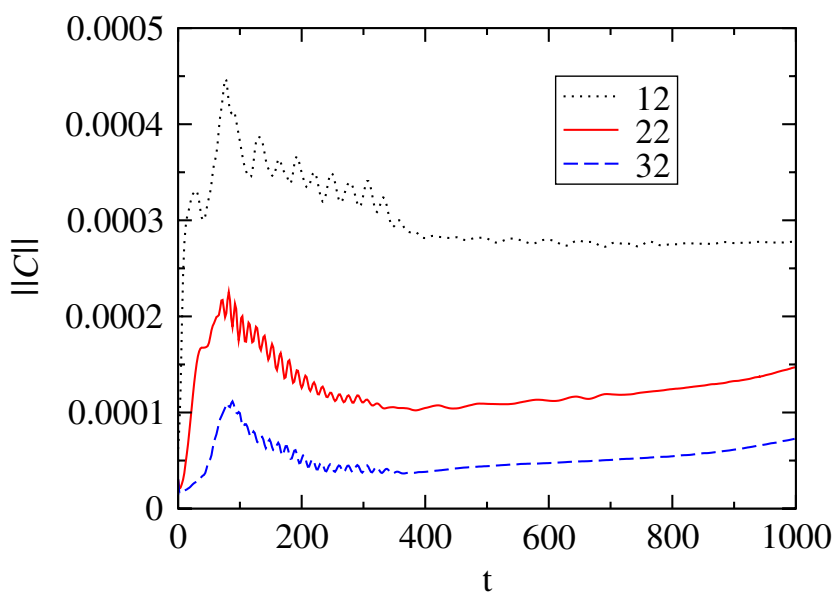

FIG. 7 (color online). The normalized constraint energy $\|\mathcal{C}\|$ defined by Eq. (71) in [39]. In this plot and all plots below, we use units in which the initial irreducible mass of the black hole is unity.

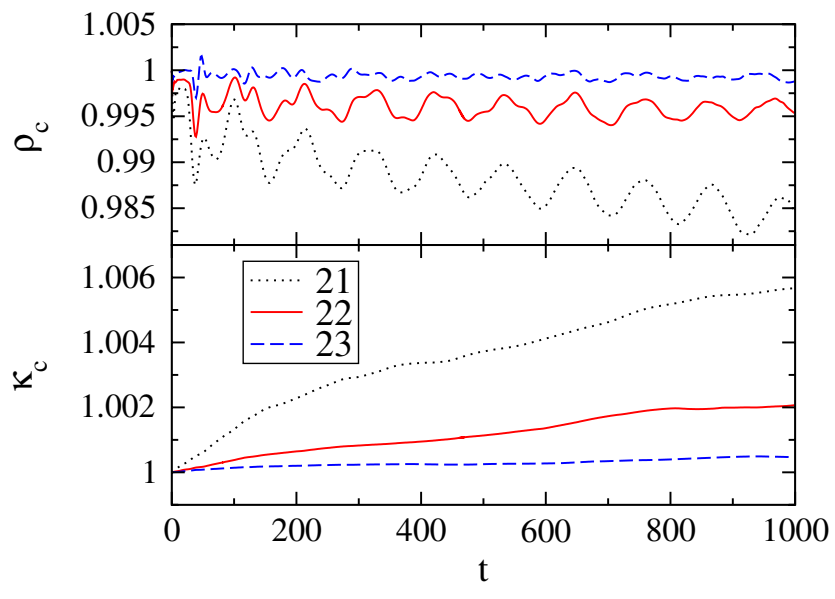

FIG. 8 (color online). The baryonic density and polytropic constant of the neutron star, normalized to their initial values.

function of time. We see that the constraints converge quickly with PS resolution. Changing the fluid grid within the range studied has a very small effect on this diagnostic, i.e. the results for Runs 21 and 23 (not plotted) would nearly coincide with that of 22. In the top panel of Fig. 8, we plot the central baryonic density normalized to its initial value. We find that the density converges with fluid grid resolution, but is insensitive to PS resolution over the range studied, i.e. Runs 12 and 32 look like 22. At the highest fluid resolution, the central density is nearly constant, with only a small downward drift, over the roughly two orbits of evolution shown. Most of the unphysical density change is caused by spurious heating. This heating can be measured by the effective polytropic constant $\kappa=$ $P / \rho^{\Gamma}$. Since there are no physical shocks during this part of the evolution, $\kappa$ should be exactly constant. We plot $\kappa$ at the center of the star, normalized to its initial value, in the bottom panel of Fig. 8. We find that the amount of spurious heating is very small, giving us confidence in our fluid evolutions.

In Fig. 9, we plot $M_{\text {irr }}$, the irreducible mass of the black hole. Perhaps surprisingly, we find that the error in $M_{\text {irr }}$ is sensitive to both grids, so to see convergence it is necessary to increase the resolutions of both grids simultaneously. Therefore, we plot $M_{\text {irr }}$ for Runs 11, 22, and 33. We see that most of the increase in black hole mass converges away. In Fig. 10, we plot the proper separation $d$ between the neutron star's center of mass and the point on the horizon on the $x$-axis facing the star. Once again, errors from both grids contribute, so one must use Runs 11, 22, and 33 to see convergence. We see that even our runs at higher resolution contain some eccentricity, presumably an artifact of the approximations, particularly the choice of zero radial velocity, used to construct the initial data. (See [72], in which we remove most of this eccentricity by adding an initial radial infall.)

These simulations can also be checked by comparing the loss of energy and angular momentum in the binary to that 


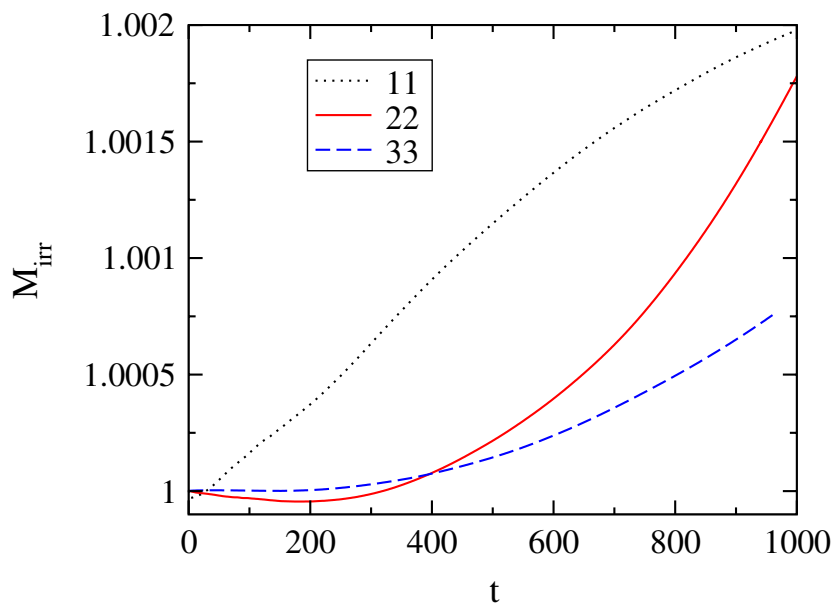

FIG. 9 (color online). The irreducible mass of the black hole.

which gravitational radiation is carrying away. In Fig. 11, we plot the total mass $M$ and angular momentum $J$ of the system as a function of time, as measured by ADM surface integrals carried out on the surface $r=300$. If carried out at future null infinity, these would be the Bondi-Sachs $M$ and $J$. (Carried out at spatial infinity, they would be the ADM $M$ and $J$.) We also monitor the gravitational wave emission via the Newman-Penrose scalar $\psi_{4}$. (See [42] for details on how $\psi_{4}$ is extracted from the evolution data.) We have varied the extraction radii and found changes below $5 \%$ (apart from retardation effects) in $M, J$, and $r \psi_{4}$ for $r$ between 200 and 400. Therefore, $M$ and $J$ should be close to their Bondi values, while $r \psi_{4}$ provides a good approximation to the asymptotic gravitational wave flux. $M$ and $J$ should decrease at a rate given by the gravitational wave flux.

After an initial burst of "junk" radiation, the true gravitational wave signal can be observed. Nearly all of the flux is carried in the $l=2, m= \pm 2$ modes. The wave signal is a sinusoid whose amplitude and frequency are nearly

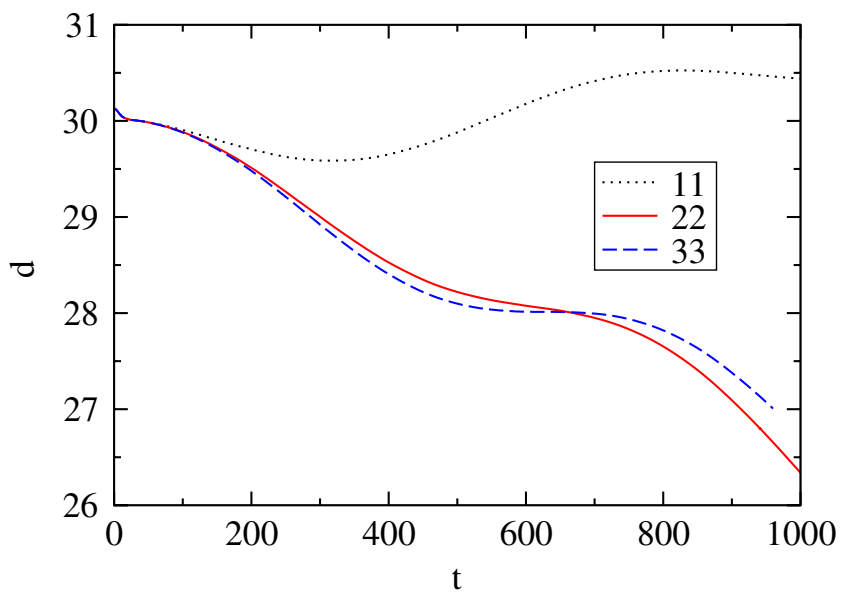

FIG. 10 (color online). The proper separation between the black hole horizon and the neutron star center.

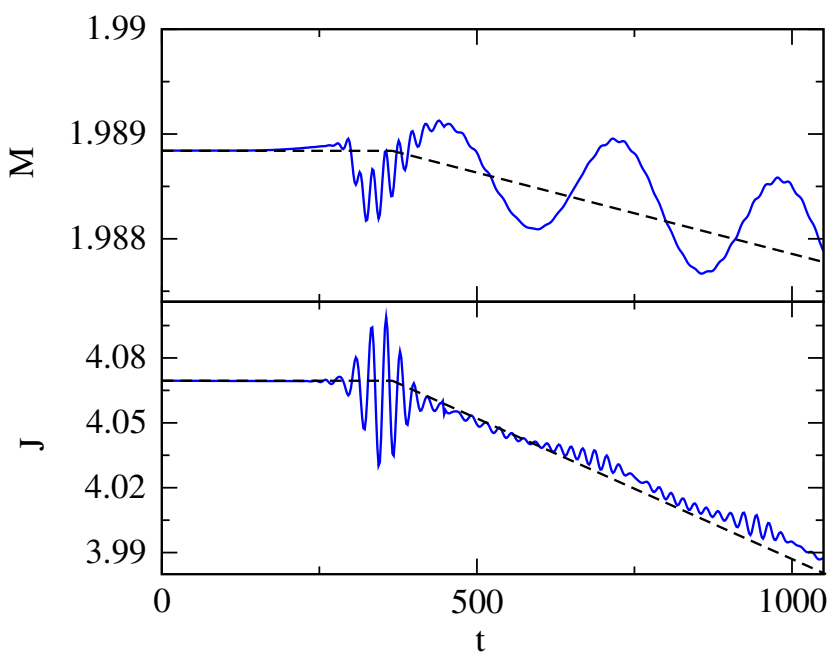

FIG. 11 (color online). The mass and angular momentum of the binary. Solid lines are ADM surface integrals. Dashed lines show the expected changes due to losses by the observed gravitational waves. $M, J$, and $r \psi_{4}$ are extracted at radius $r=$ 300 .

constant over the course of our evolution. Reading off the amplitude, frequency, and time of arrival from the $\psi_{4}$ measurements, we compute the predicted rate of mass and angular momentum loss. These are compared with the actual rates in Fig. 11, and we see that they agree well. The energy flux from a point mass binary with $M=2$ and $d=24$ is $\dot{M}=1.61 \times 10^{-6}$. The numerical flux is $\dot{M}=$ $1.55 \times 10^{-6}$, so our numerical gravitational waves are reasonable. To test the effects of the outer boundary, we reran the 22 case with outer radius at 420 and found no significant change in $M, J$, or $r \psi_{4}$. The error in $\psi_{4}$ can be estimated from the phase difference between the waves in the 22 compared to the 33 simulation, which we find to be $0.07 \mathrm{rad}$ at $t=1000$ for extraction radius $r=300$ (i.e. after a little more than two cycles).

\section{BLACK HOLE-NEUTRON STAR BINARY MERGER}

By continuing the evolution of the BHNS system, our code can simulate the merger of the two objects and determine the final stationary state. However, we find that we must introduce several modifications to our evolution techniques during the merger phase, which we find begins after about four orbits of inspiral, at around $t=$ $t_{\text {disr }} \equiv 1700$.

The most obvious adjustment to be made is in the extents of the fluid grid. When the neutron star starts to disrupt, it is no longer possible to confine this grid to a small box around the star. So we must regrid at certain times to keep the matter on the grid. Actually, regridding may also be necessary from time to time during long inspirals, because the neutron star grows in the moving coordinates as the scaling parameter $a$ in the $\mathcal{R} \mathcal{S}^{1}$ mapping decreases. 
In our simulation, we need to expand the fluid grid three times, at $t=1080, t=1540$, and $t=t_{\mathrm{diss}}$. The final fluid grid must cover a region around the neutron star and the black hole. Thus, some of the savings of our two-grid approach is lost. Even so, the fluid grid does not need to extend all the way into the wave zone, as it does in pure finite difference codes.

Crucial adjustments must also be made to the dual frame control system. During the disruption, it is no longer appropriate to fix the center of the neutron star. In a sense, the BHNS problem is easier for our code than the binary black hole problem. In the binary black hole case, the presence of two excision zones forces us to fix the locations of both holes in moving coordinates, leading to a very distorted moving coordinate system as the two holes approach each other in inertial coordinates. In the BHNS case, we can "release" the neutron star as it is disrupted, and allow it to fall into the black hole. This is done by setting $\Phi(t)=\Phi\left(t_{\mathrm{disr}}\right)$ and $a(t)=a\left(t_{\mathrm{disr}}\right)$ for $t>t_{\mathrm{disr}}$ in the $\mathcal{R S}^{2}$ mapping used to fix the star.

Because we use excision, we must continue to fix the black hole's location on our grid. However, if we were to try to do this with the $\mathcal{R} \mathcal{S}^{1}$ mapping, the parameter $a$ would approach zero as the hole moved towards the origin. Instead, we switch our coordinate control system at $t=$ $t_{\text {disr }}$. We cease evolving the $\mathcal{R} \mathcal{S}^{1}$ parameters, fixing $a(t)=$ $a\left(t_{\mathrm{disr}}\right)$ and $\Phi(t)=\Phi\left(t_{\mathrm{disr}}\right)+\dot{\Phi}\left(t_{\mathrm{disr}}\right)\left(t-t_{\mathrm{disr}}\right)$ for $t>t_{\mathrm{disr}}$. (Letting the time derivative of $\Phi$ be continuous makes the coordinate evolution smoother.) We then compose this mapping with another, a simple translation: $x^{i}=x^{\bar{i}}+C^{i}$. $C^{x}$ and $C^{y}$ are used to fix the center of the horizon.

In addition to moving, the horizon changes in size and shape, becoming much larger as it accretes matter and becoming very distorted during the merger. We find that our code becomes less accurate and less stable the deeper the PS grid extends inside the hole. Therefore, it is important to place the excision boundary as close to the apparent horizon as possible. We do this by introducing another coordinate mapping that controls the size and shape of the horizon. This mapping has the form

$$
\bar{r}=r-f(r) \sum_{l m} \lambda_{l m} Y_{l m}(\theta, \phi),
$$

where $r$ is the coordinate distance from the horizon center $(12.011,0,0), \lambda_{l m}$ are functions of time, and $f(r)$ is a smooth function of $r$. (We use a Gaussian.) The $\lambda_{l m}$ parameters are used to drive the corresponding moment of the horizon's shape in moving coordinates. For the simulation described below, the sum in Eq. (20) extends from $l=0$ to $l=6$ modes, omitting $l=1$ modes, which are controlled by the translation. At $t=t_{\text {disr }}$, we set $\lambda_{l m}=0$ for all $l>0$. $\lambda_{00}$ is set to a value that doubles the diameter of the horizon in moving coordinates, effectively increasing the grid coverage near the black hole. Because this is a discontinuous change of coordinates, we must interpolate the evolution variables onto the new grid at $t=t_{\text {disr }}$.

Finally, we find minor improvements by adjusting the generalized harmonic gauge functions $H_{a}$ during the merger. In the future, we hope to do this using dynamical gauge conditions. For now, we simply damp them to zero by setting $H_{a}(t)=H_{a}(0) e^{-\left[\left(t-t_{\text {diss }}\right) / \tau\right]^{2}}$, where $\tau=100$. Our evolutions seem to be fairly insensitive to $\tau$; it can be varied by an order of magnitude with no significant effects.

For our merger simulation, we begin at $t=1000$ with the inspiral data computed on the grids corresponding to resolution 22 and PS outer radius of 400 . We then continue the evolution of this system to later times. The evolution from $t=1000$ to $t=1700$ is very similar to the evolution from $t=0$ to $t=1000$ described in Sec. IV. At $t=1700$, matter is starting to flow off the neutron star, so we introduce the changes described above, continuing the evolution on 80 processors in order to accommodate the larger fluid grid needed. The resolution on the final fluid grid is $\Delta x=0.4$, so that there are 20 fluid grid points across the diameter of the excision zone. On the LONI Queen Bee cluster, the code must run for 43 hours (3440 CPU hours) to evolve from $t=1700$ to $t=2000$, by which time most of the matter has fallen into the hole.

Three snapshots of the neutron star density are shown in Fig. 12. A dense stream of matter flows from the star to the black hole, so that there quickly come to be two peaks in the matter density: one at the center of the neutron star and the other at the point where the stream reaches the hole. The neutron star continues to fall closer to the hole, even as it rapidly loses mass through the matter stream. The density peak corresponding to the neutron star core disappears at around $t=1800$. At around $t=1850$, the matter stream starts to close in a ring around the black hole. A shock forms at this time where matter flowing towards the hole intersects matter flowing around the hole. This can be seen in the sharp inner edge of the gray swath in the middle panel of Fig. 12. Matter falls rapidly into the hole from $t=$ 1750 until $t=2000$.

In Fig. 13, we plot the baryon rest mass $M_{0}$ on the grid together with the apparent horizon irreducible mass $M_{\text {irr }}$ as functions of time starting from $t=t_{\mathrm{disr}}$. We find that virtually all of the matter falls promptly into the black hole; $M_{0}$ drops from its initial value near unity until it stabilizes about $2 \times 10^{-4}$, indicating a very small postmerger accretion disk. This result is consistent with the small disks found by other groups (particularly Etienne et al. [36], who have also simulated the equal-mass case, but also the most recent results of Yamamoto, Shibata, and Taniguchi [35]). At $t=2200$, the matter is so sparse that we stop evolving the fluid and continue the metric evolution using our vacuum Einstein code.

The final $M_{\text {irr }}$ of the black hole is very close to 2 , and its final spin is $J / M^{2} \approx 0.7$. (The computations used to obtain the black hole spin are described in the appendices of [74].) 

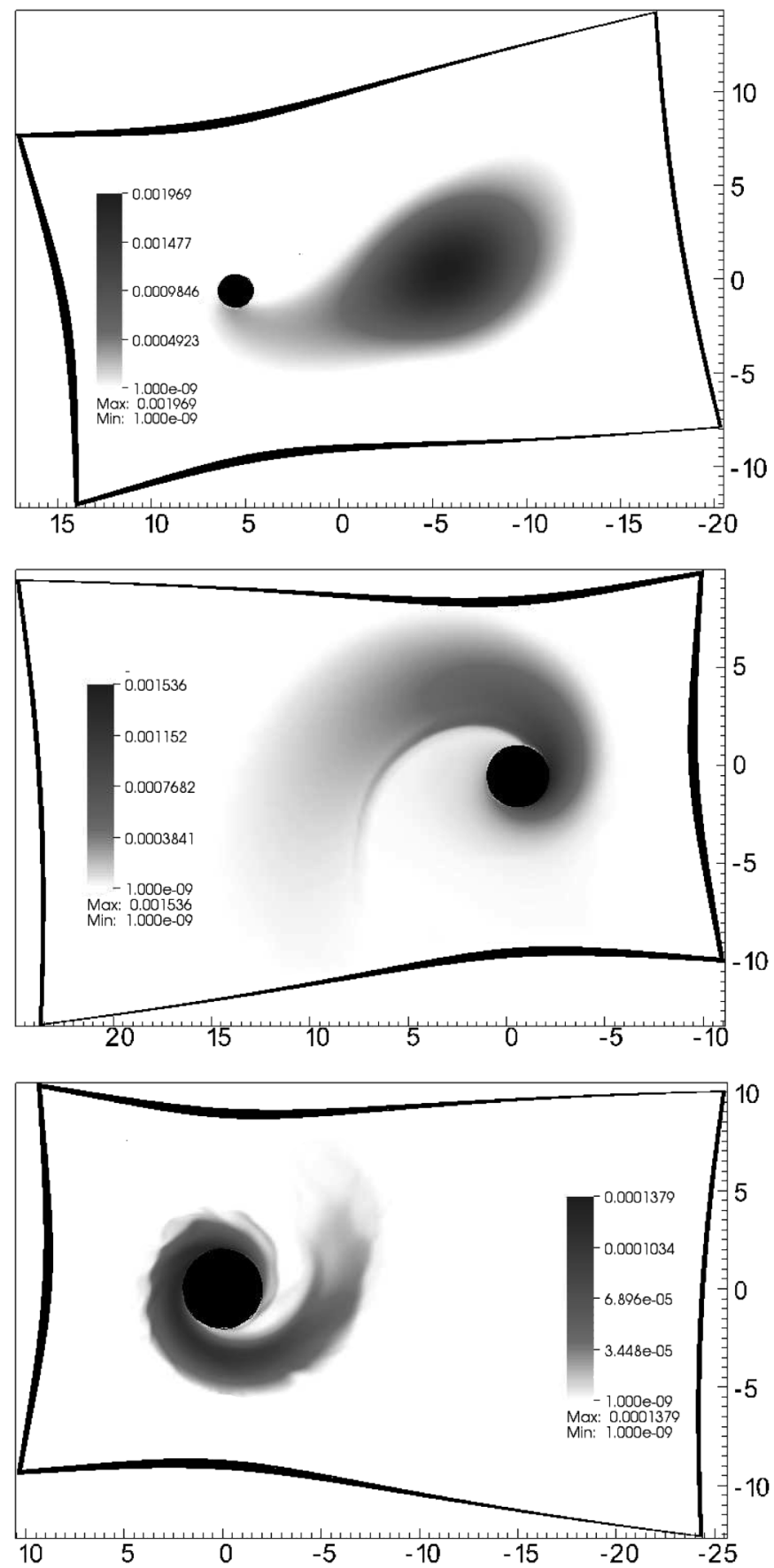

FIG. 12. Snapshots of the density and horizon shape on the equatorial plane at times $t=1730, t=1850$, and $t=1970$. Shades of gray represent $\rho / \rho_{\max }$, the density relative to its current maximum. The black object is the apparent horizon. The density and horizon are shown in inertial coordinates. The distorted rectangle is the outer edge of the fluid grid, which is a fixed rectangle in moving coordinates but moves in the inertial frame.

From $M_{\text {irr }}$ and $J$, we can compute the Christodoulou mass, and it is found to be $M_{\mathrm{c}} \approx 2.15$. That $M_{\mathrm{c}}$ is greater than 2 is presumably a result of the error in our calculation and is consistent with the observed constraint violation (see be-

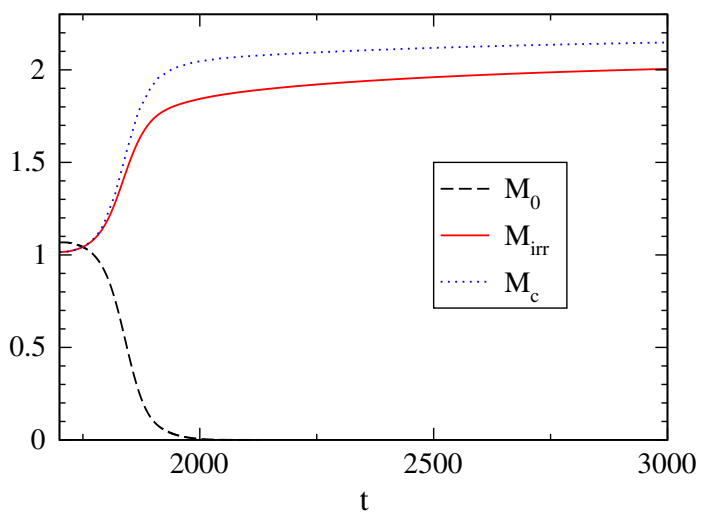

FIG. 13 (color online). The evolution of the neutron star and black hole masses from the beginning of the star's disruption to the final stationary state. Here, $M_{0}$ is the rest mass of the neutron star matter outside the hole, $M_{\text {irr }}$ is the black hole's irreducible mass, and $M_{\mathrm{c}}$ is the Christodoulou mass of the black hole, defined as $\sqrt{M_{\text {irr }}^{2}+J^{2} /\left(4 M_{\text {irr }}^{2}\right)}$, where $J$ is the black hole's spin.

low). The complete trajectories in inertial coordinates of the black hole horizon center and the neutron star center of mass are plotted in Fig. 14. In Fig. 15, we plot the $l=m=$ 2 component of $r \psi_{4}$ for the complete evolution, extracting the wave at $r=350$.

To test the stability and convergence rate of our merger algorithm, we have run the merger phase $1700<t<2050$ at a higher resolution. For this run, we increase the number of points on the fluid grid by $40 \%$ and the number of PS collocation points by one per axis per domain, keeping the extents of both grids fixed. The code runs about four times slower on the larger grid. The effects of this grid change are shown in Fig. 16. The constraint violation peaks at $t \approx$ 1900 at about $\|\mathcal{C}\|=0.1$ for the low resolution run and at

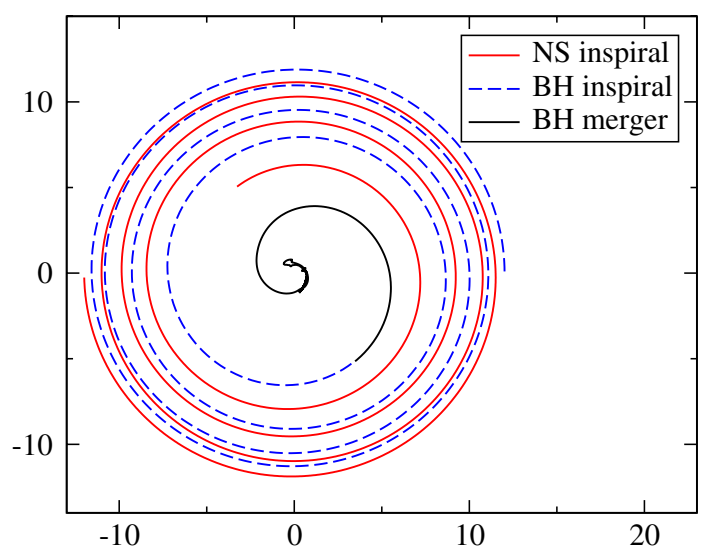

FIG. 14 (color online). The trajectory in inertial coordinates of the neutron star center from $t=0$ to $t=t_{\text {disr }}$, and that of the black hole horizon center from $t=0$ to $t=3000$. The black hole line switches from dashed to solid at $t=t_{\text {disr }}$. Both the black hole and the neutron star remain on the equatorial plane throughout the evolution. 


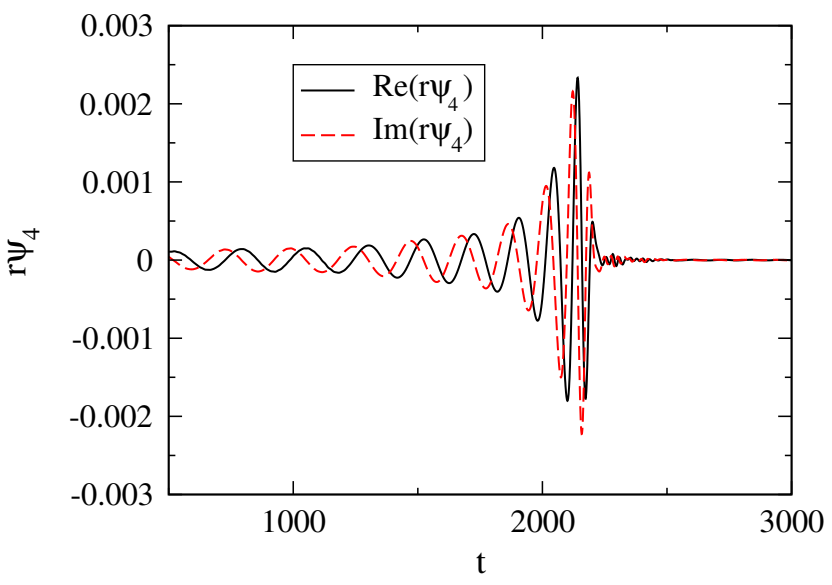

FIG. 15 (color online). The gravitational wave signal for the entire inspiral and merger (excluding the initial burst of junk radiation). The quadrupole contribution dominates, so we plot only the $l=m=2$ amplitudes.

around $\|\mathcal{C}\|=0.07$ for the high resolution run. The constraint violations decline thereafter. We note that, since the constraint energy is strongly peaked near the black hole, these numbers depend sensitively on how the norm is taken. The quantity $\|\mathcal{C}\|$, which is plotted in Fig. 16 and which is quoted above, is an L2-type norm of the form $\left(\int f^{2} d V\right)^{1 / 2}$, where $f$ represents the generalized harmonic constraints. (See Eq. 53 and Eq. 71 of [39].) If we instead use an L1-type norm of the form $\int|f| d V$, we find that this norm, when appropriately normalized, peaks at 0.02 for the low resolution run and 0.01 for the high resolution run. The Hamiltonian and momentum constraints (integrated with L1-type norms) also show viola-

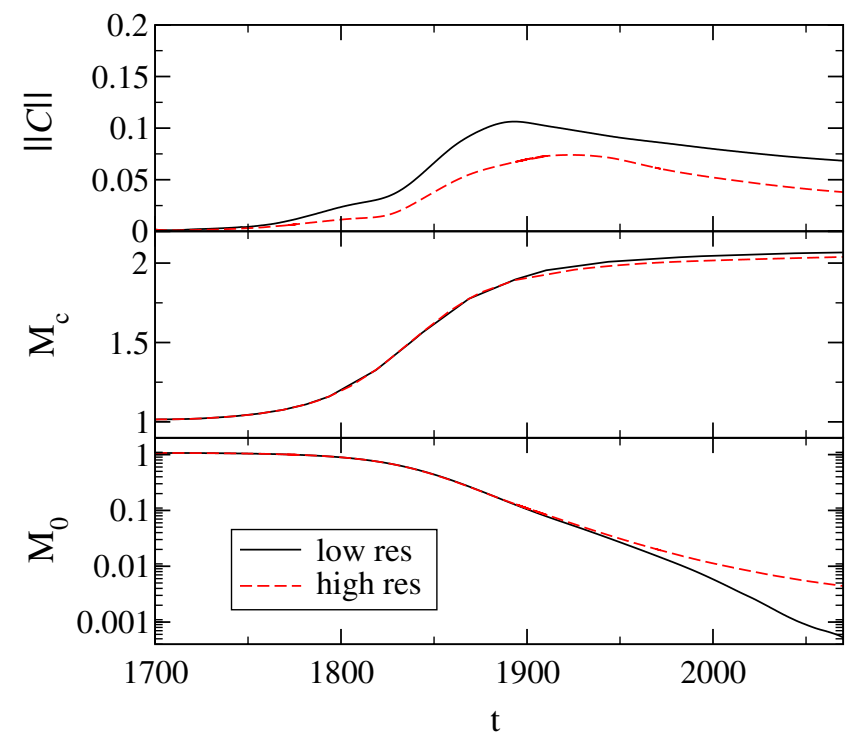

FIG. 16 (color online). The constraint energy $\|\mathcal{C}\|$, black hole mass $M_{\mathrm{c}}$, and baryonic rest mass $M_{0}$ for the merger phase in evolutions carried out at two different resolutions. tions of order $10^{-2}$. The relative $\mathrm{L} 1$ momentum constraint violation peaks at about 0.02 for the low resolution run and 0.01 for the high resolution run. The relative L1 Hamiltonian constraint violation is about 0.02 for both resolutions-convergence is not seen because this error is present in the data at $t=t_{\text {disr }}$ from which both runs start. Of course, these numbers are also very sensitive to the way the constraints are summed and normalized. The postmerger black hole mass is slightly lower at higher resolution. The accretion rate is also lower, but only after about $98 \%$ of the rest mass has already fallen into the hole. In neither run do we find a long-lived massive disk.

\section{CONCLUSIONS}

We have shown that our code can evolve inspiraling BHNS binaries with high accuracy at fairly low computational cost. This enables us to begin simulations at relatively large binary separations. Long inspirals may turn out to be very important for accurately modeling mergers; merger simulations by the Illinois group show some sensitivity to the initial separation [36], with the implication that starting too close to the merger can lead to underestimating the mass of the post-merger accretion disk. We have also shown that we can simulate the merger of these binaries, although our accuracy of these simulations is not as good so far than that of our inspirals. Also, there is every reason to believe that the techniques described here would work just as well for binary neutron stars.

An important next step is to demonstrate that our code can evolve more general BHNS binaries. We have recently begun evolving such systems with different mass ratios and black hole spins, and so far we have had little difficulty in evolving the inspirals. We hope to report on these simulations in the near future. Also, we plan to study ways to improve the accuracy of our merger simulations.

It has only been two years since the first fully relativistic BHNS merger simulations were reported, and as yet very little of the parameter space has been studied. In particular, there is a pressing need to study the effects of the black hole spin and the neutron star equation of state. There are preliminary indications (e.g. $[9,34])$ that both of these have important effects on the post-merger accretion disk mass and the gravitational wave signal. Also, both of the other groups currently performing BHNS merger simulations use very similar techniques (BSSN, moving punctures). It will be useful to compare their results with those obtained using very different techniques, like the ones reported here.

\section{ACKNOWLEDGMENTS}

We thank Francois Limousin and Manuel Tiglio for useful discussions. This work was supported in part by grants from the Sherman Fairchild Foundation to Caltech and Cornell and from the Brinson Foundation to Caltech; by NSF Grants No. PHY-0601459, No. PHY-0652995, 
No. DMS-0553302 and NASA Grant No. NNG05GG52G at Caltech; by NSF Grants No. PHY-0652952, No. DMS0553677, No. PHY-0652929, and NASA Grant No. NNG05GG51G at Cornell. This research was sup- ported in part by the NSF through TeraGrid [75] resources provided by LONI's Queen Bee cluster. Computations were also performed on Caltech's Shared Heterogeneous Cluster (SHC).
[1] A. Sadowski, K. Belczynski, T. Bulik, N. Ivanova, F. A. Rasio, and R. O'Shaughnessy, Astrophys. J. 676, 1162 (2008).

[2] R. Narayan, B. Paczynski, and T. Piran, Astrophys. J. 395, L83 (1992).

[3] N. Gehrels, C. L. Sarazin, P. T. O'Brien, B. Zhang, L. Barbier, S. D. Barthelmy, A. Blustin, D. N. Burrows, J. Cannizzo, J. R. Cummings et al., Nature (London) 437, 851 (2005).

[4] J. S. Villasenor, D. Q. Lamb, G. R. Ricker, J.-L. Atteia, N. Kawai, N. Butler, Y. Nakagawa, J. G. Jernigan, M. Boer, G. B. Crew et al., Nature (London) 437, 855 (2005).

[5] D. B. Fox, D. A. Frail, P. A. Price, S. R. Kulkarni, E. Berger, T. Piran, A. M. Soderberg, S. B. Cenko, P. B. Cameron, A. Gal-Yam et al., Nature (London) 437, 845 (2005).

[6] J. M. Lattimer and D. N. Schramm, Astrophys. J. 192, L145 (1974).

[7] S. Rosswog, Astrophys. J. 634, 1202 (2005).

[8] M. C. Miller, Astrophys. J. 626, L41 (2005).

[9] E. Rantsiou, S. Kobayashi, P. Laguna, and F. Rasio, arXiv: astro-ph/0703599 [Astrophys. J. (to be published)].

[10] M. Ruffert, H.-T. Janka, and G. Schaefer, Astron. Astrophys. 311, 532 (1996).

[11] M. Ruffert, H.-T. Janka, K. Takahashi, and G. Schaefer, Astron. Astrophys. 319, 122 (1997).

[12] S. Rosswog, M. Liebendörfer, F.-K. Thielemann, M. B. Davies, W. Benz, and T. Piran, Astron. Astrophys. 341, 499 (1999).

[13] S. Rosswog and M. B. Davies, Mon. Not. R. Astron. Soc. 334, 481 (2002).

[14] L. Dessart, C. Ott, A. Burrows, S. Rosswog, and E. Livne, arXiv:astro-ph/0806.4380.

[15] D. J. Price and S. Rosswog, Science 312, 719 (2006).

[16] R. Oechslin, H. T. Janka, and A. Marek, arXiv:astro-ph/ 0611047.

[17] M. Shibata and K. Uryū, Phys. Rev. D 61, 064001 (2000).

[18] M. Shibata and K. Uryū, Prog. Theor. Phys. 107, 265 (2002).

[19] M. Shibata, K. Taniguchi, and K. Uryū, Phys. Rev. D 68, 084020 (2003).

[20] P. Marronetti, M.D. Duez, S. L. Shapiro, and T.W. Baumgarte, Phys. Rev. Lett. 92, 141101 (2004).

[21] M. Miller, P. Gressman, and W.-M. Suen, Phys. Rev. D 69, 064026 (2004).

[22] L. Baiotti, B. Giacomazzo, and L. Rezzolla, arXiv:gr-qc/ 0804.0594 [Phys. Rev. D (to be published)]

[23] M. Shibata, K. Taniguchi, and K. Uryū, Phys. Rev. D 71, 084021 (2005).

[24] M. Shibata and K. Taniguchi, Phys. Rev. D 73, 064027 (2006).
[25] M. Anderson, E.W. Hirschmann, L. Lehner, S. L. Liebling, P. M. Motl, D. Neilsen, C. Palenzuela, and J. E. Tohline, Phys. Rev. Lett. 100, 191101 (2008).

[26] Y. T. Liu, S. L. Shapiro, Z. B. Etienne, and K. Taniguchi, Phys. Rev. D 78, 024012 (2008).

[27] W. H. Lee and W.Ł. Kluźniak, Mon. Not. R. Astron. Soc. 308, 780 (1999).

[28] W.H. Lee and W.Ł. Kluźniak, Astrophys. J. 526, 178 (1999).

[29] H.-T. Janka, T. Eberl, M. Ruffert, and C. L. Fryer, Astrophys. J. Lett. 527, L39 (1999).

[30] S. Rosswog, R. Speith, and G. A. Wynn, Mon. Not. R. Astron. Soc. 351, 1121 (2004).

[31] J. A. Faber, T. W. Baumgarte, S. L. Shapiro, K. Taniguchi, and F. A. Rasio, Phys. Rev. D 73, 024012 (2006).

[32] F. Löffler, L. Rezzolla, and M. Ansorg, Phys. Rev. D 74, 104018 (2006).

[33] M. Shibata and K. Uryū, Classical Quantum Gravity 24, S125 (2007).

[34] M. Shibata and K. Taniguchi, Phys. Rev. D 77, 084015 (2008).

[35] T. Yamamoto, M. Shibata, and K. Taniguchi, Phys. Rev. D 78, 064054 (2008)

[36] Z. B. Etienne, J.A. Faber, Y. T. Liu, S. L. Shapiro, K. Taniguchi, and T.W. Baumgarte, Phys. Rev. D 77, 084002 (2008).

[37] L. E. Kidder, M. A. Scheel, S. A. Teukolsky, E. D. Carlson, and G. B. Cook, Phys. Rev. D 62, 084032 (2000).

[38] M. A. Scheel, L.E. Kidder, L. Lindblom, H. P. Pfeiffer, and S. A. Teukolsky, Phys. Rev. D 66, 124005 (2002).

[39] L. Lindblom, M. A. Scheel, L. E. Kidder, R. Owen, and O. Rinne, Classical Quantum Gravity 23, S447 (2006).

[40] M. Boyle, L. Lindblom, H. Pfeiffer, M. Scheel, and L. E. Kidder, Phys. Rev. D 75, 024006 (2007).

[41] M. A. Scheel et al., Phys. Rev. D 74, 104006 (2006).

[42] H. P. Pfeiffer et al., Classical Quantum Gravity 24, S59 (2007).

[43] M. Boyle et al., Phys. Rev. D 76, 124038 (2007).

[44] E. Tadmor, SIAM J. Numer. Anal. 26, 30 (1989).

[45] W. S. Don, J. Comput. Phys. 110, 103 (1994).

[46] H. Dimmelmeier, J. Novak, J. A. Font, J. M. Ibáñez, and E. Müller, Phys. Rev. D 71, 064023 (2005).

[47] H. Dimmelmeier, P. Cerda-Duran, and A. Marek, AIP Conf. Proc. 861, 596 (2006).

[48] C. D. Ott et al., Phys. Rev. Lett. 98, 261101 (2007).

[49] H. Dimmelmeier, C. D. Ott, H.-T. Janka, A. Marek, and E. Mueller, Phys. Rev. Lett. 98, 251101 (2007).

[50] P. Cerda-Duran, J. A. Font, and H. Dimmelmeier, arXiv: astro-ph/0703597.

[51] H. Friedrich, Commun. Math. Phys. 100, 525 (1985).

[52] F. Pretorius, Classical Quantum Gravity 22, 425 (2005). 
[53] L. Lehner, O. Reula, and M. Tiglio, Classical Quantum Gravity 22, 5283 (2005).

[54] T. Matsushima and P. S. Marcus, J. Comput. Phys. 120, 365 (1995).

[55] O. Rinne, L. Lindblom, and M.A. Scheel, Classical Quantum Gravity 24, 4053 (2007).

[56] J. P. Boyd, J. Comput. Phys. 103, 243 (1992).

[57] J. A. Font, T. Goodale, S. Iyer, M. Miller, L. Rezzolla, E. Seidel, N. Stergioulas, W.-M. Suen, and M. Tobias, Phys. Rev. D 65, 084024 (2002).

[58] L. Baiotti, I. Hawke, P. J. Montero, F. Löffler, L. Rezzolla, N. Stergioulas, J. A. Font, and E. Seidel, Phys. Rev. D 71, 024035 (2005).

[59] P. Colella and P. R. Woodward, J. Comput. Phys. 54, 174 (1984).

[60] X.-D. Liu and S. Osher, J. Comput. Phys. 142, 304 (1998).

[61] T. C. Xu-Dong Liu and Stanley Osher, J. Comput. Phys. 115, 200 (1994).

[62] B. v. L. A. Harten and P. D. Lax, SIAM Rev. 25, 35 (1983).

[63] L. Del Zanna and N. Bucciantini, Astron. Astrophys.. 390, 1177 (2002).

[64] I. Hawke, F. Loffler, and A. Nerozzi, Phys. Rev. D 71,
104006 (2005).

[65] F. C. Michel, Astrophys. Space Sci. 15, 153 (1972).

[66] L. I. Petrich, S. L. Shapiro, and S. A. Teukolsky, Phys. Rev. Lett. 60, 1781 (1988).

[67] G. B. Cook, S. L. Shapiro, and S. A. Teukolsky, Astrophys. J. 398, 203 (1992).

[68] J. L. Friedman, J. R. Ipser, and R. D. Sorkin, Astrophys. J. 325, 722 (1988).

[69] T.W. Baumgarte, S.L. Shapiro, and M. Shibata, Astrophys. J. 528, L29 (2000).

[70] M. D. Duez, Y. T. Liu, S. L. Shapiro, and B. C. Stephens, Phys. Rev. D 72, 024028 (2005).

[71] F. Douchin and P. Haensel, Astron. Astrophys. 380, 151 (2001).

[72] F. Foucart, L.E. Kidder, H. P. Pfeiffer, and S. A. Teukolsky, Phys. Rev. D 77, 124051 (2008).

[73] M. Caudill, G. B. Cook, J. D. Grigsby, and H. P. Pfeiffer, Phys. Rev. D 74, 064011 (2006).

[74] G. Lovelace, R. Owen, H. P. Pfeiffer, and T. Chu, Phys. Rev. D 78, 084017 (2008).

[75] C. Catlett et al., in Advances in Parallel Computing, edited by L. Grandinetti (IOS Press, Amsterdam, 2007). 